\title{
THE CASSELMAN-SHALIKA FORMULA FOR A DISTINGUISHED MODEL
}

\author{
WILLIAM D. BANKS
}

(Communicated by William Adams)

\begin{abstract}
Unramified Whittaker functions and their analogues occur naturally in number theory as terms in the Fourier expansions of automorphic forms. Precise information about these functions is useful in many aspects of study, such as in the construction of L-functions. In this paper, the method of Casselman and Shalika is used to derive explicit values for the analogue of the unramified Whittaker function in a distinguished model that arises in connection with quadratic base change.
\end{abstract}

\section{INTRODUCTION}

The unramified Whittaker functions and their analogues play an important role in modern number theory, arising naturally as terms in the Fourier expansions of automorphic forms. It is generally desirable (whenever possible) to calculate explicit values for these functions, as the information proves useful in many aspects of study related to the automorphic form (for example, in the construction of associated L-functions). When an automorphic representation possesses a Whittaker model or another suitable unique model, the method described in [3] may be used to compute an explicit formula (the CasselmanShalika formula) for the values of the unramified Whittaker function (or the analogous function).

In this paper, we consider the following distinguished model that arises in connection with quadratic base change and the theory of the Asai L-function (see [1], [5], and [6]). The existence and uniqueness of this model was established by Hakim in [4]. Let $F$ be a nonarchimedean local field of characteristic zero and with odd residue characteristic, and let $q_{F}$ denote the cardinality of the residue field. Let $E / F$ be an unramified quadratic extension of $F$. Let $\mathscr{O}_{E}$ denote the ring of integers in $E$. Put $G_{E}=G L(2, E), G_{F}=G L(2, F)$, and $K_{E}=G L\left(2, \mathscr{O}_{E}\right)$. If $(\pi, V)$ is an irreducible, admissible, unramified principal series representation of $G_{E}$ that is trivial on the center $Z_{E}$ of $G_{E}$, it is known that:

(i) There exists a nonzero spherical vector $\phi^{0} \in V$, unique up to complex constant, such that $\pi(k) \phi^{0}=\phi^{0}$ for all $k \in K_{E}$.

Received by the editors September 3, 1991 and, in revised form, May 28, 1993.

1991 Mathematics Subject Classification. Primary 11F70, 22 E35. 
(ii) There exists a nonzero linear functional $T: V \rightarrow \mathbb{C}$, unique up to complex constant, such that $T(\pi(g) \phi)=T(\phi)$ for all $g \in G_{F}$ and $\phi \in V$ (see [4]).

As $\pi$ is in the principal series, $\phi^{0}$ may be regarded as a complex-valued function on $G_{E}$. In $\S 6$, it is shown that $T\left(\phi^{0}\right) \neq 0$. Thus, we can normalize the constants in (i) and (ii) so that $\phi^{0}=1$ on $K_{E}$, and $T\left(\phi^{0}\right)=1$. Now let $\mathscr{Q}$ be the vector space of all locally-constant complex-valued functions on $G_{E}$ that satisfy $f\left(g g^{\prime}\right)=f\left(g^{\prime}\right)$ for all $g \in G_{F}$ and $g^{\prime} \in G_{E}$. The group $G_{E}$ acts on $\mathscr{Q}$ by right translation. Let $\mathscr{Q}(\pi)$ denote the invariant subspace of $\mathscr{Q}$ spanned by functions $Q_{\phi}$ of the form $Q_{\phi}(g)=T(\pi(g) \phi)$. The map $\phi \mapsto Q_{\phi}$ yields an isomorphism of $G_{E}$-modules $V \cong \mathscr{Q}(\pi)$, and $\mathscr{Q}(\pi)$ is called the distinguished model for $V$.

For the above model, the correct analogue of the unramified Whittaker function is the function $Q_{0}=Q_{\phi^{0}}$ given by $Q_{0}(g)=T\left(\pi(g) \phi^{0}\right)$ for all $g \in G_{E}$. Observe that $Q_{0}$ is a well-defined function on the double cosets of $G_{F} \backslash G_{E} / K_{E}$, hence it suffices to compute $Q_{0}$ on the complete set of double coset representatives $\left\{M_{k}: k=0,1,2, \ldots\right\}$ described in $\S 3$. The main result of this paper (proved in $\S 6$ ) is the following:

Theorem. Let $t_{\chi} \in G L(2, \mathbb{C})$ be the Satake parameter of $\pi$, and let $\vee^{k}$ be the $k$ th symmetric tensor representation of $G L(2, \mathbb{C})$ for each $k \geq 0$. Then:

$$
\begin{aligned}
& Q_{0}\left(M_{0}\right)=1, \\
& Q_{0}\left(M_{1}\right)=q_{F}^{-1}\left(q_{F}-1\right)^{-1}\left(q_{F} \operatorname{Tr}\left(t_{\chi}\right)-\left(q_{F}+1\right)\right),
\end{aligned}
$$

and if $k \geq 2$

$$
Q_{0}\left(M_{k}\right)=q_{F}^{-k}\left(q_{F}-1\right)^{-1}\left(q_{F} \operatorname{Tr}\left(\vee^{k} t_{\chi}\right)-\left(q_{F}+1\right) \operatorname{Tr}\left(\vee^{k-1} t_{\chi}\right)+\operatorname{Tr}\left(\vee^{k-2} t_{\chi}\right)\right) .
$$

\section{SOME NOTATION}

Let $v$ be a fixed valuation of $E$ (which restricts to a valuation of $F$ ), and fix once and for all a prime element $\varpi$ in $F$ such that $v(\varpi)=1$ (this is possible since $E / F$ is unramified).

Let $\mathscr{O}_{E}$ denote the ring of integers of $E, \wp_{E}$ its unique maximal ideal, and $\mathscr{O}_{E}^{\times}$its group of units. Let $q_{E}$ be the (odd) cardinality of the residue field $\mathscr{O}_{E} / \wp_{E}$, and let $|\cdot|_{E}=q_{E}^{-v(\cdot)}$ be the absolute value symbol for $E$ corresponding to $v$. We similarly define $\mathscr{O}_{F}, \wp_{F}, \mathscr{O}_{F}^{\times}, q_{F}$, and $|\cdot|_{F}=q_{F}^{-v(\cdot)}$. Then $q_{E}=q_{F}^{2}$ and $|\cdot|_{E}=|\cdot|_{F}^{2}$.

As $E / F$ is quadratic, $E=F(\mu)$ for some $\mu \in \mathscr{O}_{E}^{\times}-\mathscr{O}_{F}^{\times}$with $\mu^{2} \in \mathscr{O}_{F}^{\times}$. Then $E=F \oplus \mu F$ and $\mathscr{O}_{E}=\mathscr{O}_{F} \oplus \mu \mathscr{O}_{F}$. By Hensel's lemma, the image of $\tau=\mu^{2}$ in the residue field $\mathscr{O}_{F} / \wp_{F}$ is a quadratic nonresidue.

For $G=G L(2)$, let $P$ be the standard Borel subgroup of $G$, let $N$ be the unipotent radical of $P$, and let $Z$ be the center of $G$. The Weyl group $W$ of $G$ consists of the elements $\left\{e, w_{0}\right\}$, where $e=\left({ }_{1}{ }_{1}\right)$ and $w_{0}=\left({ }_{1}{ }^{1}\right)$. Once and for all, fix $\xi=\left(\begin{array}{c}1 \mu \\ 1\end{array}\right) \in G_{E}$.

An additive Haar measure $d x$ on $F$ is said to be normalized if $\operatorname{vol}\left(\mathscr{O}_{F}\right)=$ 1. A multiplicative Haar measure $d^{\times} y$ on $F^{\times}$is said to be normalized if $d^{\times} y=|y|_{F}^{-1} d y$ for some normalized additive Haar measure $d y$ on $F$. By the 
Bruhat decomposition, $G_{F}=P_{F} \cup P_{F} w_{0} P_{F}$. Then $Z_{F} \backslash P_{F}$ has measure 0 in $Z_{F} \backslash G_{F}$ and the matrices

$$
\left\{\left(\begin{array}{cc}
y & x \\
& 1
\end{array}\right) w_{0}\left(\begin{array}{cc}
1 & \bar{x} \\
& 1
\end{array}\right): x, \bar{x} \in F \text { and } y \in F^{\times}\right\}
$$

form a complete set of distinct representatives for $Z_{F} \backslash P_{F} w_{0} P_{F}$. A left Haar measure $d g$ is said to be normalized if

$$
\int_{Z_{F} \backslash G_{F}} \phi(g) d g=\iint_{F} \int_{F^{\times}} \phi\left(\left(\begin{array}{ll}
y & x \\
& 1
\end{array}\right) w_{0}\left(\begin{array}{ll}
1 & \bar{x} \\
& 1
\end{array}\right)\right) \frac{d x d^{\times} y}{|y|_{F}} d \bar{x}
$$

for all $\phi$ integrable on $Z_{F} \backslash G_{F}$, where $d x$ and $d \bar{x}$ are normalized additive Haar measures for $F$, and $d^{\times} y$ is a normalized multiplicative Haar measure for $F^{\times}$.

\section{Double COSET DECOMPOSITION}

The function $Q_{0}$ described in $\S 1$ is well defined on $G_{F} \backslash G_{E} / K_{E}$, since

$$
Q_{0}\left(g g^{\prime} k\right)=T\left(\pi\left(g g^{\prime} k\right) \phi^{0}\right)=T\left(\pi(g) \pi\left(g^{\prime}\right) \pi(k) \phi^{0}\right)=T\left(\pi\left(g^{\prime}\right) \phi^{0}\right)=Q_{0}\left(g^{\prime}\right)
$$

for all $g \in G_{F}, g^{\prime} \in G_{E}$, and $k \in K_{E}$. Hence, it suffices to determine $Q_{0}$ on a complete set of double coset representatives for $G_{F} \backslash G_{E} / K_{E}$.

By the Iwasawa decomposition, a complete set of double coset representatives for $N_{E} \backslash G_{E} / K_{E}$ is given by the matrices $\mathscr{A}=\left\{\left(\varpi^{k} \varpi^{l}\right): k, l \in \mathbb{Z}\right\}$. Then $N_{E} \mathscr{A}$ is a complete set of representatives for $G_{E} / K_{E}$. As $N_{E} \mathscr{A}=\mathscr{A} N_{E}$ and $\mathscr{A} \subset G_{F}$, it follows that $N_{E}$ is a complete set of representatives for $G_{F} \backslash G_{E} / K_{E}$. To reduce the set further, note that each $z \in E$ may be written in the form $z=x+u \varpi^{-k} \mu$, where $x \in F, u \in \mathscr{O}_{F}^{\times}$, and $k \in \mathbb{Z}$. Then

$$
\left(\begin{array}{cc}
1 & z \\
& 1
\end{array}\right)=\left(\begin{array}{ll}
u \varpi^{-k} & x \\
& 1
\end{array}\right)\left(\begin{array}{ll}
\varpi^{k} & \mu \\
& 1
\end{array}\right)\left(\begin{array}{ll}
u^{-1} & \\
& 1
\end{array}\right) \text {, }
$$

with $\left(\begin{array}{c}u \varpi^{-k} x \\ 1\end{array}\right) \in G_{F}$ and $\left(u^{-1}{ }_{1}\right) \in K_{E}$. Moreover, since $\left(\varpi^{k} \mu_{1}\right) \in G_{F} K_{E}$ whenever $k \leq 0$, it follows that the matrices $\left\{M_{k}=\left(\varpi^{k} \mu\right): k \in \mathbb{Z}, k \geq 0\right\}$ form a complete set of double coset representatives for $G_{F} \backslash G_{E} / K_{E}$. Moreover, it may be shown that these matrices represent distinct cosets.

\section{THE LINEAR FUNCTIONAL $T$}

As a principal series representation, the space $V=V_{\chi}$ of $\pi$ is the vector space $\operatorname{Ind}_{P_{E}}^{G_{E}}\left(\delta^{\frac{1}{2}} \chi\right)$ consisting of all locally-constant complex-valued functions on $G_{E}$ that satisfy $\phi\left(p g^{\prime}\right)=\left(\delta^{\frac{1}{2}} \chi\right)(p) \phi\left(g^{\prime}\right)$ for all $p \in P_{E}$ and $g^{\prime} \in G_{E}$. Here $\chi$ and $\delta$ are characters of $P_{E}$ :

$$
\begin{aligned}
& \chi\left(\begin{array}{cc}
y_{1} & * \\
& y_{2}
\end{array}\right)=\chi_{1}\left(y_{1}\right) \chi_{2}\left(y_{2}\right), \\
& \delta\left(\begin{array}{cc}
y_{1} & * \\
& y_{2}
\end{array}\right)=\left|\frac{y_{1}}{y_{2}}\right|_{E}=q_{E}^{v\left(y_{2}\right)-v\left(y_{1}\right)}
\end{aligned}
$$


for all $y_{1}, y_{2} \in E^{\times}$and $* \in E$, where $\chi_{1}, \chi_{2}$ are characters of $E^{\times}$. Since $\pi$ is unramified, $\chi$ is unramified, and we can assume that

$$
\chi_{1}(y)=\alpha^{v(y)}, \quad \chi_{2}(y)=\beta^{v(y)}
$$

for some $\alpha, \beta \in \mathbb{C}^{\times}$and all $y \in E^{\times}$. Moreover, the condition that $\pi$ is trivial on $Z_{E}$ implies $\alpha \beta=1$. Thus,

$$
\phi\left(\left(\begin{array}{cc}
y_{1} & * \\
& y_{2}
\end{array}\right) g\right)=\left(\beta q_{F}\right)^{v\left(y_{2}\right)-v\left(y_{1}\right)} \phi(g)
$$

for all $y_{1}, y_{2} \in E^{\times}, * \in E, g \in G$, and $\phi \in V_{\chi}$.

An unramified character $\chi$ of $P_{E}$ as above is said to dominant if $|\beta|>1$.

Proposition 1. Suppose that $\chi$ is a dominant character and $d g$ is a normalized left Haar measure on $Z_{F} \backslash G_{F}$. Then the integral

$$
\tilde{T}(\phi)=\int_{Z_{F} \backslash G_{F}} \phi\left(w_{0} \xi g\right) d g
$$

is well defined and absolutely convergent for all $\phi \in V_{\chi}$, and therefore defines a linear functional $\tilde{T}: V_{\chi} \rightarrow \mathbb{C}$ such that

$$
\widetilde{T}(\pi(g) \phi)=\widetilde{T}(\phi)
$$

for all $g \in G_{F}$ and $\phi \in V_{\chi}$.

Proof. The only issue here is that of convergence. By uniqueness of Haar measure, the integral $\widetilde{T}(\phi)$ is a nonzero multiple of the integral:

$$
\int_{K_{F}} \int_{F^{\times}} \int_{F} \phi\left(w_{0} \xi\left(\begin{array}{cc}
y & x \\
& 1
\end{array}\right) k\right) \frac{d x d^{\times} y}{|y|_{F}} d k,
$$

where $d k$ is a left Haar measure on $K_{F}=G L\left(2, \mathscr{O}_{F}\right), d x$ is a normalized additive Haar measure for $F$, and $d^{\times} y$ is a normalized multiplicative Haar measure for $F^{\times}$.

For each $k \in K_{F}$ and $\phi \in V_{\chi}$, let

$$
\mathscr{G}_{\phi}(k)=\int_{F^{\times}} \int_{F} \phi\left(w_{0} \xi\left(\begin{array}{cc}
y & x \\
& 1
\end{array}\right) k\right) \frac{d x d^{\times} y}{|y|_{F}} .
$$

Then each $\mathscr{G}_{\phi}$ is locally-constant on $K_{F}$ (since $\phi$ is locally-constant). As $K_{F}$ is compact, the absolute convergence of $\widetilde{T}(\phi)$ follows from the absolute convergence of the integrals $\mathscr{G}_{\phi}(k)$. Moreover, there is no loss of generality in 
assuming that $k=e$, since $\mathscr{G}_{\phi}(k)=\mathscr{G}_{\pi(k) \phi}(e)$. Thus,

$$
\begin{aligned}
\mathscr{G}_{\phi}(e) & =\int_{F^{\times}} \int_{F} \phi\left(w_{0} \xi\left(\begin{array}{rr}
y & x \\
1
\end{array}\right)\right) \frac{d x d^{\times} y}{|y|_{F}} \\
& =\int_{F^{\times}} \int_{F} \phi\left(w_{0} \xi\left(\begin{array}{ll}
y^{-1} & x \\
1
\end{array}\right)\right)|y|_{F} d x d^{\times} y \\
& =\int_{F-\{0\}} \int_{F} \phi\left(w_{0} \xi\left(\begin{array}{cc}
y^{-1} & x \\
1
\end{array}\right)\right) d x d y \\
& =\int_{F-\{0\}} \int_{F}\left(\beta q_{F}\right)^{-v(y)} \phi\left(\begin{array}{cc}
\left.w_{0}\left(\begin{array}{cc}
1 & x y+y \mu \\
1
\end{array}\right)\right) d x d y \\
\end{array}\right. \\
& =\int_{F-\{0\}} \int_{F} \beta^{-v(y)} \phi\left(w_{0}\left(\begin{array}{cc}
1 & x+y \mu \\
1
\end{array}\right)\right) d x d y \\
& =\int_{F} \int_{F-\{0\}} \beta^{-v(y)} \phi\left(w_{0}\left(\begin{array}{cc}
1 & x+y \mu \\
1
\end{array}\right)\right) d y d x
\end{aligned}
$$

by Fubini's theorem. Since $\chi$ is dominant $(|\beta|>1)$, we can add the point $y=0$ to each inner integral to obtain

$$
\begin{aligned}
\mathscr{G}_{\phi}(e) & =\int_{F} \int_{F} \beta^{-v(y)} \phi\left(w_{0}\left(\begin{array}{cc}
1 & x+y \mu \\
1
\end{array}\right)\right) d y d x \\
& =\int_{E} \beta^{-v(\operatorname{Im}(z))_{\phi}}\left(w_{0}\left(\begin{array}{ll}
1 & z \\
& 1
\end{array}\right)\right) d z,
\end{aligned}
$$

where $\operatorname{Im}(z)=y$ if $z=x+y \mu$, and $d z=d y d x$ is a normalized additive Haar measure for $E$. Since $\phi$ is locally constant, we can choose $L \gg 0$ so that $\phi\left(w_{0}\left(\begin{array}{cc}1 & z \\ 1\end{array}\right)\right)=\phi\left(w_{0}\right)$ if $v(z) \geq L$, and $\phi\left(\begin{array}{c}1_{z^{-1}} \\ 1\end{array}\right)=\phi(e)$ if $v(z) \leq-L$. Also whenever $z \neq 0$ we have

$$
\begin{aligned}
\phi\left(w_{0}\left(\begin{array}{ll}
1 & z \\
& 1
\end{array}\right)\right) & =\phi\left(\left(\begin{array}{cc}
1 & z^{-1} \\
& 1
\end{array}\right)\left(\begin{array}{cc}
-z^{-1} & \\
& z
\end{array}\right)\left(\begin{array}{cc}
1 & \\
z^{-1} & 1
\end{array}\right)\right) \\
& =\left(\beta q_{F}\right)^{2 v(z)} \phi\left(\begin{array}{cc}
1 & \\
z^{-1} & 1
\end{array}\right) .
\end{aligned}
$$

It follows that $\mathscr{G}_{\phi}(e)$ can be expressed as a sum of three integrals:

$$
\begin{aligned}
\phi\left(w_{0}\right) & \int_{\substack{E \\
v(z) \geq L}} \beta^{-v(\operatorname{Im}(z))} d z+\phi(e) \int_{\substack{E \\
v(z) \leq-L}} \beta^{-v(\operatorname{Im}(z))}\left(\beta q_{F}\right)^{2 v(z)} d z \\
& +\int_{-L<v(z)<L} \beta^{-v(\operatorname{Im}(z))} \phi\left(w_{0}\left(\begin{array}{rr}
1 & z \\
& 1
\end{array}\right)\right) d z .
\end{aligned}
$$

The first two integrals converge absolutely (by a straightforward calculation) whenever $|\beta|>1$, and the last integral converges absolutely for all $\beta$ since it is the integral of a continuous function over a compact region. 
For dominant characters $\chi$, the lemma in $\S 6$ shows that $\widetilde{T}\left(\phi^{0}\right) \neq 0$, and we can define

$$
T(\phi)=\frac{\widetilde{T}(\phi)}{\widetilde{T}\left(\phi^{0}\right)}
$$

for $\phi \in V_{\chi}$. Then $T: V_{\chi} \rightarrow \mathbb{C}$ is the unique linear functional that satisfies $T(\pi(g) \phi)=T(\phi)$ for all $g \in G_{F}, \phi \in V_{\chi}$, and $T\left(\phi^{0}\right)=1$.

A flat section is a collection $\left\{\phi_{\chi} \in \operatorname{Ind}_{P_{E}}^{G_{E}}\left(\delta^{\frac{1}{2}} \chi\right): \chi\right.$ unramified $\}$ such that $\left.\phi_{\chi}\right|_{K_{E}}$ is independent of $\chi$. It follows from the Iwasawa decomposition that every flat section $\left\{\phi_{\chi}\right\}$ is equicontinuous; that is, there exists an open neighborhood $K_{\phi}$ of the identity, which is independent of $\chi$, such that $\phi_{\chi}$ is $K_{\phi}$-fixed for every $\chi$.

Proposition 2. Let $g^{\prime}$ be a fixed element of $G_{E}$, and let $\left\{\phi_{\chi}\right\}$ be a flat section. Then the function $\left(1-\left(\beta q_{F}\right)^{-2}\right) T\left(\pi\left(g^{\prime}\right) \phi_{\chi}\right)$, initially defined for dominant characters $\chi$ (i.e., $|\beta|>1)$, extends to an entire function of $\beta \in \mathbb{C}$.

Proof. From the proof of Proposition 1 (when $\chi$ is dominant)

$$
\begin{aligned}
& \mathscr{G}_{\pi\left(g^{\prime}\right) \phi_{\chi}}(k)=\phi_{\chi}\left(w_{0} k g^{\prime}\right) \int_{\substack{E \\
v(z) \geq L_{\chi}(k)}} \beta^{-v(\operatorname{Im}(z))} d z \\
& +\phi_{\chi}\left(k g^{\prime}\right) \int_{E} \beta^{-v(\operatorname{Im}(z))}\left(\beta q_{F}\right)^{2 v(z)} d z \\
& +\int_{-L_{\chi}(k)<v(z)<L_{\chi}(k)} \beta^{-v(\operatorname{Im}(z))} \phi_{\chi}\left(w_{0}\left(\begin{array}{ll}
1 & z \\
& 1
\end{array}\right) k g^{\prime}\right) d z,
\end{aligned}
$$

for each unramified character $\chi$ and $k \in K_{F}$, where $L_{\chi}(k) \gg 0$ is chosen so that

$$
\begin{aligned}
& v(z) \geq L_{\chi}(k) \Longrightarrow \phi_{\chi}\left(w_{0}\left(\begin{array}{ll}
1 & z \\
& 1
\end{array}\right) k g^{\prime}\right)=\phi_{\chi}\left(w_{0} k g^{\prime}\right), \\
& v(z) \leq-L_{\chi}(k) \Longrightarrow \phi_{\chi}\left(\left(\begin{array}{cc}
1 & \\
z^{-1} & 1
\end{array}\right) k g^{\prime}\right)=\phi_{\chi}\left(k g^{\prime}\right) .
\end{aligned}
$$

As $\left\{\phi_{\chi}\right\}$ is equicontinuous, we can choose $L_{\chi}(k)=L(k)$ independent of $\chi$ and locally-constant on $K_{F}$. As $K_{F}$ is compact, we can choose $L(k)=L$ independent of $k$.

The first two integrals in (1) may be directly computed for dominant $\chi$ :

$$
\begin{gathered}
\phi\left(w_{0} k g^{\prime}\right) \int_{v(z) \geq L} \beta^{-v(\operatorname{Im}(z))} d z=\phi\left(w_{0} k g^{\prime}\right) \frac{\left(\beta q_{F}^{2}\right)^{-L}}{1-\left(\beta q_{F}\right)^{-1}} \\
\phi_{\chi}\left(k g^{\prime}\right) \int_{v(z) \leq-L} \beta^{-v(\operatorname{Im}(z))}\left(\beta q_{F}\right)^{2 v(z)} d z \\
=\phi_{\chi}\left(k g^{\prime}\right) \frac{\beta^{-L}\left(1-q_{F}^{-1}\right)\left(1-\left(\beta q_{F}^{2}\right)^{-1}\right)}{\left(1-\left(\beta q_{F}\right)^{-1}\right)\left(1-\beta^{-1}\right)}
\end{gathered}
$$

As before, the last integral in (1) converges absolutely for all unramified characters $\chi$ and all $k \in K_{F}$ and consequently defines an entire function of $\beta$. 
It follows that for each $k \in K_{F},\left(1-\left(\beta q_{F}\right)^{-1}\right)\left(1-\beta^{-1}\right) \mathscr{G}_{\pi\left(g^{\prime}\right) \phi_{x}}(k)$ continues to an entire function of $\beta$, and as $K_{F}$ is compact, it follows that $\left(1-\left(\beta q_{F}\right)^{-1}\right)\left(1-\beta^{-1}\right) \widetilde{T}\left(\pi\left(g^{\prime}\right) \phi_{\chi}\right)$ has this continuation as well. For each unramified character $\chi$, let $\phi_{\chi}^{0}$ be the unique $K_{E}$-fixed vector in $\operatorname{Ind}_{P_{E}}^{G_{E}}\left(\delta^{\frac{1}{2}} \chi\right)$ such that $\phi_{\chi}^{0}=1$ on $K_{E}$. Then the collection $\left\{\phi_{\chi}^{0}\right\}$ is a flat section. The lemma in $\S 6$ shows that

$$
\widetilde{T}\left(\phi_{\chi}^{0}\right)=\frac{\left(1-q_{F}^{-2}\right)\left(1+\left(\beta q_{F}\right)^{-1}\right)}{\left(1-\beta^{-1}\right)}
$$

for dominant $\chi$, hence:

$$
\left(1-\left(\beta q_{F}\right)^{-2}\right) T\left(\pi\left(g^{\prime}\right) \phi_{\chi}\right)=\frac{\left(1-\left(\beta q_{F}\right)^{-1}\right)\left(1-\beta^{-1}\right) \widetilde{T}\left(\pi\left(g^{\prime}\right) \phi_{\chi}\right)}{\left(1-q_{F}^{-2}\right)} .
$$

This equation implies the proposition.

\section{IWAHORI FIXED VECTORS}

Let $B_{E}$ be the Iwahori subgroup of $G_{E}$ (i.e., the inverse image of the standard Borel subgroup under the natural homomorphism $\left.K_{E} \rightarrow G L\left(2, \mathscr{O}_{E} / \wp_{E}\right)\right)$. Let $V_{\chi}^{B_{E}}$ be the space of Iwahori fixed vectors:

$$
V_{\chi}^{B_{E}}=\left\{\phi \in V_{\chi}: \pi(b) \phi=\phi \text { for all } b \in B_{E}\right\} .
$$

By the Iwahori factorization, each element $g^{\prime} \in G_{E}$ may be expressed in the form $g^{\prime}=p \bar{w}_{g^{\prime}} b$ where $p \in P_{E}, \bar{w}_{g^{\prime}} \in W$, and $b \in B_{E}$. Moreover, $\bar{w}_{g^{\prime}}$ is uniquely determined by $g^{\prime}$. For each $w \in W$, let

$$
\phi_{w, \chi}\left(g^{\prime}\right)= \begin{cases}\left(\delta^{\frac{1}{2}} \chi\right)(p) & \text { if } g^{\prime}=p \bar{w}_{g^{\prime}} b \text { and } \bar{w}_{g^{\prime}}=w, \\ 0 & \text { otherwise. }\end{cases}
$$

By a theorem of Casselman (see [2]), the vectors $\left\{\phi_{w, \chi}\right\}_{w \in W}$ form a basis (the Casselman basis) for $V_{X}^{B_{E}}$.

Let $N\left(\mathscr{O}_{E}\right)=N_{E} \cap K_{E}$, and let $d n$ be a left Haar measure on $N_{E}$ such that $\operatorname{vol}\left(N\left(\mathscr{O}_{E}\right)\right)=1$. For each $k \geq 0$ and $g^{\prime} \in G_{E}$, the integral

$$
F_{k}\left(g^{\prime}\right)=\int_{N\left(\mathscr{O}_{E}\right)} \phi_{\chi}^{0}\left(g^{\prime} n\left(\begin{array}{ll}
\varpi^{k} & \\
& 1
\end{array}\right)\right) d n
$$

converges absolutely for all unramified $\chi$. Note that $F_{0}=\phi_{\chi}^{0}$. It is known that the $\left\{F_{k}\right\}$ are Iwahori fixed vectors given explicitly by

$$
F_{k}=\sum_{w \in W} c_{w}(\chi)\left(\delta^{\frac{1}{2} w} \chi\right)\left(\begin{array}{ll}
\varpi^{k} & \\
& 1
\end{array}\right) \phi_{w, \chi}
$$

where $c_{e}(\chi)=1, c_{w_{0}}(\chi)=\left(1-\left(\beta q_{F}\right)^{-2}\right)\left(1-\beta^{-2}\right)^{-1}$, and ${ }^{w} \chi$ is the character given by

$$
{ }^{w} \chi\left(\begin{array}{cc}
y_{1} & * \\
& y_{2}
\end{array}\right)=\chi\left(w^{-1}\left(\begin{array}{ll}
y_{1} & \\
& y_{2}
\end{array}\right) w\right)
$$

for all $y_{1}, y_{2} \in E^{\times}$and $* \in E$. 
Proposition 3. For dominant $\chi, Q_{0}\left(M_{0}\right)=T\left(F_{0}\right)$, and if $k \geq 1$,

$$
Q_{0}\left(M_{k}\right)=\left(1-q_{F}^{-1}\right)^{-1} T\left(F_{k}-q_{F}^{-1} F_{k-1}\right) .
$$

Proof. We compute:

$$
\begin{aligned}
& \widetilde{T}\left(F_{k}\right)=\int_{Z_{F} \backslash G_{F}} F_{k}\left(w_{0} \xi g\right) d g \\
& =\int_{Z_{F} \backslash G_{F}} \int_{N\left(\mathcal{O}_{E}\right)} \phi_{\chi}^{0}\left(w_{0} \xi g n\left(\begin{array}{ll}
\varpi^{k} & \\
& 1
\end{array}\right)\right) d n d g \\
& =\int_{Z_{F} \backslash G_{F}} \int_{\mathscr{O}_{F}} \int_{\mathscr{O}_{F}} \phi_{\chi}^{0}\left(w_{0} \xi g\left(\begin{array}{ll}
1 & y \\
& 1
\end{array}\right)\left(\begin{array}{cc}
1 & x \mu \\
& 1
\end{array}\right)\left(\begin{array}{ll}
\varpi^{k} & \\
& 1
\end{array}\right)\right) d y d x d g \\
& =\int_{Z_{F} \backslash G_{F}} \int_{\mathcal{Q}_{F}} \phi_{\chi}^{0}\left(w_{0} \xi g\left(\begin{array}{cc}
\varpi^{k} & x \mu \\
& 1
\end{array}\right)\right) d x d g
\end{aligned}
$$

since $\left(\begin{array}{c}1 \\ y \\ 1\end{array}\right) \in G_{F}$ for all $y \in \mathscr{O}_{F}$ and $\operatorname{vol}\left(\mathscr{O}_{F}\right)=1$.

If $x=u \varpi^{n}$ with $u \in \mathscr{O}_{F}^{\times}, n \in \mathbb{Z}$, then $\left(u \varpi_{1}^{n}\right) \in G_{F},\left(u_{1}^{-1}\right) \in K_{E}$, and therefore,

$$
\begin{aligned}
& \int_{Z_{F} \backslash G_{F}} \phi_{\chi}^{0}\left(w_{0} \xi g\left(\begin{array}{cc}
\varpi^{k} & x \mu \\
& 1
\end{array}\right)\right) d g
\end{aligned}
$$

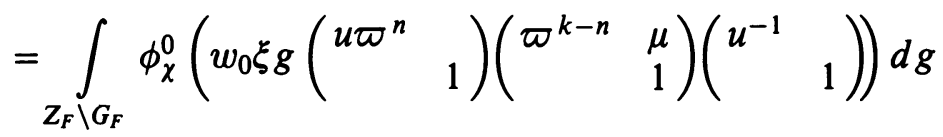

$$
\begin{aligned}
& =\int_{Z_{F} \backslash G_{F}} \phi_{\chi}^{0}\left(w_{0} \xi g\left(\varpi^{k-n} \begin{array}{c}
\mu \\
\end{array}\right)\right) d g \\
& =\widetilde{T}\left(\pi\left(\begin{array}{ll}
\varpi^{k-n} & \mu \\
& 1
\end{array}\right) \phi_{\chi}^{0}\right)= \begin{cases}\tilde{T}\left(\phi_{\chi}^{0}\right) Q_{0}\left(M_{0}\right) & \text { if } n \geq k, \\
\widetilde{T}\left(\phi_{\chi}^{0}\right) Q_{0}\left(M_{k-n}\right) & \text { if } n<k .\end{cases}
\end{aligned}
$$

Thus, applying Fubini's theorem to (5), it follows that

$$
\begin{aligned}
\tilde{T}\left(F_{k}\right)= & \sum_{n \geq 0} \int_{v(x)=n} \phi_{\chi}^{0}\left(w_{0} \xi g\left(\begin{array}{cc}
\varpi^{k-n} & x \mu \\
1
\end{array}\right)\right) d g d x \\
= & \sum_{n \geq k} \operatorname{vol}\left(\wp_{F}^{n}-\wp_{F}^{n+1}\right) \tilde{T}\left(\phi_{\chi}^{0}\right) Q_{0}\left(M_{0}\right) \\
& +\sum_{n=0}^{k-1} \operatorname{vol}\left(\wp_{F}^{n}-\wp_{F}^{n+1}\right) \tilde{T}\left(\phi_{\chi}^{0}\right) Q_{0}\left(M_{k-n}\right) \\
= & q_{F}^{-k} \widetilde{T}\left(\phi_{\chi}^{0}\right) Q_{0}\left(M_{0}\right)+\left(1-q_{F}^{-1}\right) \widetilde{T}\left(\phi_{\chi}^{0}\right) \sum_{n=0}^{k-1} q_{F}^{-n} Q_{0}\left(M_{k-n}\right) .
\end{aligned}
$$

The proposition now follows from this by a simple inductive argument. 


\section{Calculation of $T\left(\phi_{w, \chi}\right)$ and $Q_{0}\left(M_{k}\right)$}

Lemma. For dominant $\chi$, we have

$$
\widetilde{T}\left(\phi_{\chi}^{0}\right)=\frac{\left(1-q_{F}^{-2}\right)\left(1+\left(\beta q_{F}\right)^{-1}\right)}{\left(1-\beta^{-1}\right)} .
$$

Proof. The left Haar measure $d g$ is normalized (see $\S 2$ ) so that:

$$
\begin{aligned}
\tilde{T}\left(\phi_{\chi}^{0}\right) & =\int_{Z_{F} \backslash G_{F}} \phi_{\chi}^{0}\left(w_{0} \xi g\right) d g \\
& =\int_{F} \int_{F^{\times}} \int_{F} \phi_{\chi}^{0}\left(w_{0} \xi\left(\begin{array}{cc}
y & x \\
& 1
\end{array}\right) w_{0}\left(\begin{array}{cc}
1 & \bar{x} \\
& 1
\end{array}\right)\right) \frac{d x d^{\times} y}{|y|_{F}} d \bar{x} .
\end{aligned}
$$

If $\bar{x} \in \mathscr{O}_{F}$, then $w_{0}\left(\begin{array}{r}1 \\ 1\end{array}\right) \in K_{F}$ and

$$
\begin{gathered}
\int_{F^{\times}} \int_{F} \phi_{\chi}^{0}\left(w_{0} \xi\left(\begin{array}{ll}
y & x \\
& 1
\end{array}\right) w_{0}\left(\begin{array}{ll}
1 & \bar{x} \\
1
\end{array}\right)\right) \frac{d x d^{\times} y}{|y|_{F}} \\
=\int_{F^{\times}} \int_{F} \phi_{\chi}^{0}\left(w_{0} \xi\left(\begin{array}{cc}
y & x \\
& 1
\end{array}\right)\right) \frac{d x d^{\times} y}{|y|_{F}} .
\end{gathered}
$$

On the other hand, if $\bar{x} \notin \mathscr{O}_{F}$, then $\left(\begin{array}{c}1 \\ x^{-1}\end{array}\right) \in K_{F}$ and

$$
\begin{aligned}
& \int_{F^{\times}} \int_{F} \phi_{\chi}^{0}\left(w_{0} \xi\left(\begin{array}{cc}
y & x \\
& 1
\end{array}\right) w_{0}\left(\begin{array}{cc}
1 & \bar{x} \\
& 1
\end{array}\right)\right) \frac{d x d^{\times} y}{|y|_{F}} \\
& =\int_{F^{\times}} \int_{F} \phi_{\chi}^{0}\left(w_{0} \xi\left(\begin{array}{cc}
y & x \\
& 1
\end{array}\right)\left(\begin{array}{cc}
1 & \bar{x}^{-1} \\
& 1
\end{array}\right)\left(\begin{array}{cc}
-\bar{x}^{-1} & \\
& \bar{x}
\end{array}\right)\left(\begin{array}{cc}
1 & \\
\bar{x}^{-1} & 1
\end{array}\right)\right) \frac{d x d^{\times} y}{|y|_{F}} \\
& =\int_{F^{\times}} \int_{F} \phi_{\chi}^{0}\left(w_{0} \xi\left(\begin{array}{cc}
-y \bar{x}^{-2} & x+y \bar{x}^{-1} \\
1
\end{array}\right)\right) \frac{d x d^{\times} y}{|y|_{F}} \\
& =|\bar{x}|_{F}^{-2} \int_{F^{\times}} \int_{F} \phi_{\chi}^{0}\left(w_{0} \xi\left(\begin{array}{cc}
y & x \\
& 1
\end{array}\right)\right) \frac{d x d^{\times} y}{|y|_{F}} .
\end{aligned}
$$

Thus,

$$
\begin{aligned}
\widetilde{T}\left(\phi_{\chi}^{0}\right) & =\int_{F} q_{F}^{\min (2 v(x), 0)} d \bar{x} \int_{F^{\times}} \int_{F} \phi_{\chi}^{0}\left(w_{0} \xi\left(\begin{array}{ll}
y & x \\
1
\end{array}\right)\right) \frac{d x d^{\times} y}{|y|_{F}} \\
& =\left(1+q_{F}^{-1}\right) \mathscr{G}_{\phi_{\chi}^{0}}(e) .
\end{aligned}
$$

Since $\phi_{\chi}^{0}$ is $K_{E}$-fixed, it follows from equation (1) of $\S 4$ that

$$
\mathscr{G}_{\phi_{\chi}^{0}}(e)=\int_{\substack{E \\ v(z) \leq-1}} \beta^{-v(\operatorname{Im}(z))}\left(\beta q_{F}\right)^{2 v(z)} d z+\int_{\substack{E \\ v(z) \geq 0}} \beta^{-v(\operatorname{Im}(z))} d z .
$$

To evaluate these integrals, take $L=0, \phi=\phi_{\chi}^{0}$ in equation (2) of $\S 4$, and $L=1, \phi=\phi_{\chi}^{0}$ in equation (3) of $\S 4$. The lemma follows. 
We now turn to the calculation of $\tilde{T}\left(\phi_{w, \chi}\right)$ for $w \in W$. For this, it will be necessary to determine those $g^{\prime}=w_{0} \xi\left(\begin{array}{cc}y & x \\ 1\end{array}\right) w_{0}\left(\begin{array}{c}1 x \\ 1\end{array}\right)$ in $G$ such that $\bar{w}_{g^{\prime}}=w_{0}$. Thus, let $\lambda=y^{-1}(x+\mu)$ and $\eta=\lambda^{-1}+\bar{x}=y(x-\mu)\left(x^{2}-\tau\right)^{-1}+\bar{x}$, where $\tau=\mu^{2}$. Then

$$
\begin{aligned}
w_{0} \xi\left(\begin{array}{cc}
y & x \\
& 1
\end{array}\right) w_{0}\left(\begin{array}{cc}
1 & \bar{x} \\
& 1
\end{array}\right) & =\left(\begin{array}{ll}
1 & \\
& y
\end{array}\right)\left(\begin{array}{ll}
1 & \\
\lambda & 1
\end{array}\right)\left(\begin{array}{cc}
1 & \bar{x} \\
& 1
\end{array}\right) \\
& =\left(\begin{array}{ll}
1 & \\
& y
\end{array}\right)\left(\begin{array}{cc}
1 & \lambda^{-1} \\
& 1
\end{array}\right)\left(\begin{array}{cc}
-\lambda^{-1} & \\
& \lambda
\end{array}\right) w_{0}\left(\begin{array}{cc}
1 & \eta \\
& 1
\end{array}\right) .
\end{aligned}
$$

It follows that $\bar{w}_{g^{\prime}}=w_{0}$ if and only if $\eta \in \mathscr{O}_{E}$ and hence, if and only if $y\left(x^{2}-\tau\right)^{-1} \in \mathscr{O}_{F}$ and $\bar{x}+y x\left(x^{2}-\tau\right)^{-1} \in \mathscr{O}_{F}$. Consequently (if $\bar{w}_{g^{\prime}}=w_{0}$ ),

$$
\phi_{w_{0}, \chi}\left(g^{\prime}\right)=\left(\beta q_{F}\right)^{v\left(y \lambda^{2}\right)}=\left(\beta q_{F}\right)^{(\min (2 v(x), 0)-v(y))} .
$$

Proposition 4. For dominant $\chi$, we have

$$
T\left(\phi_{e, \chi}\right)=\frac{\left(\beta q_{F}\right)^{-1}-\beta^{-2}}{1-\beta^{-2}} \text { and } T\left(\phi_{w_{0}, \chi}\right)=\frac{1}{1+\left(\beta q_{F}\right)^{-1}} .
$$

Proof. In this proof, Fubini's theorem will be applied frequently without mention.

We compute

$$
\begin{aligned}
\widetilde{T}\left(\phi_{w_{0}, \chi}\right) & =\int_{F} \int_{F^{\times}} \int_{F} \phi_{w_{0}, \chi}\left(w_{0} \xi\left(\begin{array}{cc}
y & x \\
1
\end{array}\right) w_{0}\left(\begin{array}{cc}
1 & \bar{x} \\
1
\end{array}\right)\right) \frac{d x d^{\times} y}{|y|_{F}} d \bar{x} \\
& =\int_{F} \int_{F} \int_{F^{\times}}\left(\beta q_{F}\right) \\
& \left.(\min (2 v(x), \mu)-v(y)) \frac{d^{\times} y d x}{|y|_{F}} d \bar{x} . \tau\right)^{-1+x \in \mathcal{O}_{E}}
\end{aligned}
$$

We apply a change of variables $y \mapsto\left(x^{2}-\tau\right) y_{o}, d^{\times} y \mapsto d^{\times} y_{o}$, where $y_{o} \in$ $\mathscr{O}_{F}-\{0\}$. Because the image of $\tau$ in $\mathscr{O}_{F} / \wp_{F}$ is a quadratic nonresidue (see $\S 2$ ), it follows that $v(y)=\min (2 v(x), 0)+v\left(y_{o}\right)$, and thus

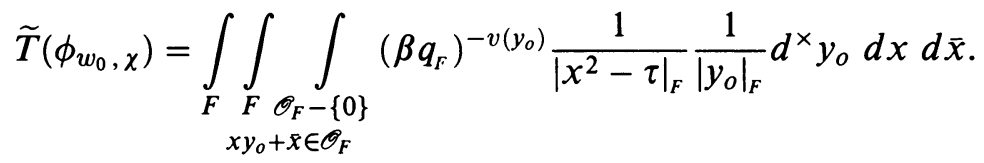

Next, we apply a change of variables $x \mapsto y_{o}^{-1}\left(x_{o}-\bar{x}\right), d x \mapsto\left|y_{o}\right|_{F} d x_{o}$, where $x_{o} \in \mathscr{O}_{F}:$

$$
\begin{aligned}
\tilde{T}\left(\phi_{w_{0}, \chi}\right) & =\int_{F} \int_{\mathscr{O}_{F}-\{0\}} \int_{\mathscr{O}_{F}}\left(\beta q_{F}\right)^{-v\left(y_{o}\right)} \frac{1}{\left.\left|\left(y_{o}^{-1}\left(x_{o}-\bar{x}\right)\right)^{2}-\tau\right|\right|_{F}} \frac{1}{\left|y_{o}\right|_{F}^{2}} d x_{o} d^{\times} y_{o} d \bar{x} \\
& =\int_{\mathscr{O}_{F}-\{0\}} \int_{\mathscr{O}_{F}} \beta^{-v\left(y_{o}\right)} q_{F}^{v\left(y_{o}\right)}\left(\int_{F} \frac{d \bar{x}}{\left|\left(y_{o}^{-1}\left(x_{o}-\bar{x}\right)\right)^{2}-\tau\right|_{F}}\right) d x_{o} d^{\times} y_{o} .
\end{aligned}
$$

To compute the inner integral, we apply a change of variables $\bar{x} \mapsto y_{o} \bar{x}_{o}+x_{o}$, 
$d \bar{x} \mapsto\left|y_{o}\right|_{F} d \bar{x}_{o}:$

$$
\begin{aligned}
\int_{F} \frac{d \bar{x}}{\left|\left(y_{o}^{-1}\left(x_{o}-\bar{x}\right)\right)^{2}-\tau\right|_{F}} & =\left|y_{o}\right|_{F} \int_{F} \frac{d \bar{x}_{o}}{\left|\bar{x}_{o}^{2}-\tau\right|_{F}} \\
& =\left|y_{o}\right|_{F} \int_{F} q_{F}^{\min \left(2 v\left(\bar{x}_{o}\right), 0\right)} d \bar{x}_{o}=\left|y_{o}\right|_{F}\left(1+q_{F}^{-1}\right)
\end{aligned}
$$

Then

$$
\tilde{T}\left(\phi_{w_{0}, \chi}\right)=\left(1+q_{F}^{-1}\right) \int_{\mathscr{O}_{F}-\{0\}} \int_{\mathscr{O}_{F}} \beta^{-v\left(y_{o}\right)} d x_{o} d^{\times} y_{o}=\frac{\left(1-q_{F}^{-2}\right)}{\left(1-\beta^{-1}\right)},
$$

and therefore

$$
T\left(\phi_{w_{0}, \chi}\right)=\frac{\tilde{T}\left(\phi_{w_{0}, \chi}\right)}{\widetilde{T}\left(\phi_{\chi}^{0}\right)}=\frac{1}{1+\left(\beta q_{F}\right)^{-1}} .
$$

From $\S 4$, we know that $F_{0}=\phi_{\chi}^{0}$; thus, using equation (4) we can write

$$
\phi_{\chi}^{0}=c_{e}(\chi) \phi_{e, \chi}+c_{w_{0}}(\chi) \phi_{w_{0}, \chi}
$$

Applying the linear functional $T$, we obtain

$$
\begin{aligned}
1 & =c_{e}(\chi) T\left(\phi_{e, \chi}\right)+c_{w_{0}}(\chi) T\left(\phi_{w_{0}, \chi}\right) \\
& =T\left(\phi_{e, \chi}\right)+\frac{1+\left(\beta q_{F}\right)^{-1}}{1-\beta^{-2}},
\end{aligned}
$$

whence the proposition follows.

Proof of Theorem. By Proposition 2, each $Q_{0}\left(M_{k}\right)$ (regarded as a function of $\beta$ ) continues meromorphically to the entire complex plane, with possible (simple) poles at $\pm q_{F}^{-1}$.

Applying the linear functional $T$ to equation (4), we have by Proposition 4

$$
\begin{aligned}
T\left(F_{k}\right) & =\sum_{w \in W} c_{w}(\chi)\left(\delta^{\frac{1}{2} w} \chi\right)\left(\begin{array}{ll}
\varpi^{k} & \\
1
\end{array}\right) T\left(\phi_{w, \chi}\right) \\
& =q_{F}^{-k} \beta^{-k} \frac{\left(\beta q_{F}\right)^{-1}-\beta^{-2}}{1-\beta^{-2}}+\frac{1-\left(\beta q_{F}\right)^{-2}}{1-\beta^{-2}} q_{F}^{-k} \beta^{k} \frac{1}{1+\left(\beta q_{F}\right)^{-1}} .
\end{aligned}
$$

Substituting $\alpha=\beta^{-1}$, this simplifies to

$$
\begin{aligned}
T\left(F_{k}\right) & =q_{F}^{-k}\left(\alpha^{k} \frac{\alpha-q_{F}^{-1}}{\alpha-\beta}-\beta^{k} \frac{\beta-q_{F}^{-1}}{\alpha-\beta}\right) \\
& =q_{F}^{-k}\left(\frac{\alpha^{k+1}-\beta^{k+1}}{\alpha-\beta}-q_{F}^{-1} \frac{\alpha^{k}-\beta^{k}}{\alpha-\beta}\right) \\
& =q_{F}^{-k}\left(S_{k}(\alpha, \beta)-q_{F}^{-1} S_{k-1}(\alpha, \beta)\right),
\end{aligned}
$$

where $S_{k}(\alpha, \beta)$ is defined for $k \in \mathbb{Z}$ by

$$
S_{k}(\alpha, \beta)= \begin{cases}\sum_{i=0}^{k} \alpha^{i} \beta^{k-i} & \text { if } k \geq 0 \\ 0 & \text { otherwise }\end{cases}
$$


Note that each $S_{k}(\alpha, \beta)$ is an entire function of $\beta$.

Now, by Proposition 3 (when $\chi$ is dominant), $Q_{0}\left(M_{0}\right)=T\left(F_{0}\right)=1$, and when $k \geq 1$ :

$$
\begin{aligned}
Q_{0}\left(M_{k}\right) & =\left(1-q_{F}^{-1}\right)^{-1} T\left(F_{k}-q_{F}^{-1} F_{k-1}\right)=\left(1-q_{F}^{-1}\right)^{-1}\left(T\left(F_{k}\right)-q_{F}^{-1} T\left(F_{k-1}\right)\right) \\
& =q_{F}^{-k}\left(q_{F}-1\right)^{-1}\left(q_{F} S_{k}(\alpha, \beta)-\left(q_{F}+1\right) S_{k-1}(\alpha, \beta)+S_{k-2}(\alpha, \beta)\right) .
\end{aligned}
$$

Thus, $Q_{0}\left(M_{k}\right)$ continues to an entire function of $\beta$ for each $k$. Bearing in mind that the Satake parameter for $\pi$ is the matrix $t_{\chi}=\left({ }^{\alpha}{ }_{\beta}\right) \in G L(2, \mathbb{C})$, then $\operatorname{Tr}\left(\vee^{k} t_{\chi}\right)=S_{k}(\alpha, \beta)$ for each $k \geq 0$, whence the theorem is established.

\section{ACKNOWLEDGMENT}

The author would like to thank Daniel Bump for many valuable discussions and helpful suggestions, and the referee for his careful review and insightful comments on the text.

\section{REFERENCES}

1. T. Asai, On certain Dirichlet series associated with Hilbert modular forms and Rankin's method, Math. Ann. 226 (1977), 81-94.

2. W. Casselman, The unramified principal series of p-adic groups I: the spherical function, Compositio Math. 40 (1980), 387-406.

3. W. Casselman and J. Shalika, The unramified principal series of p-adic groups II: the Whittaker function, Compositio Math. 41 (1980), 207-231.

4. J. Hakim, Distinguished p-adic representations, Duke Math. J. 62 (1991), 1-22.

5. G. Harder, R. P. Langlands, and M. Rapoport, Algebraische Zyklen auf Hilbert-BlumenthalFlächen, Crelles J. 366 (1986), 53-120.

6. Y. Ye, Kloosterman integrals and base change for GL(2), Crelles J. 400 (1989), 57-121.

Department of Mathematics, Stanford University, Stanford, California 94305-2125

E-mail address: banks@cauchy.stanford.edu 